\title{
Extensive Corneal Neovascularisation Treatment by Ultraviolet Corneal Collagen Crosslinking
}

\author{
Mohammad M Shehadeh, ${ }^{1,3}$ Mohammad T Akkawi, ${ }^{1}$ Vasilios F Diakonis, ${ }^{2,3}$ Ammar A Aghbar ${ }^{1}$ and \\ Abdelraheem Abu Shanab \\ 1. Faculty of Medicine and Health Sciences, An-Najah National University Hospital, An-Najah National University, Nablus, Palestine; 2. Bascom \\ Palmer Eye Institute, Miller School of Medicine, University of Miami, Miami, Florida, US; 3. Vardinoyannion Eye Institute of Crete (VEIC), Faculty \\ of Medicine, University of Crete, Heraklion, Crete, Greece
}

DOI: https://doi.org/10.17925/EOR.2017.11.01.62

$\mathrm{T}$

he purpose of this article is to report a case of extensive corneal neovascularisation that was treated by ultraviolet corneal collagen crosslinking (CXL). The case report is about a 24-year-old man who was referred to the cornea clinic with a case of keratitis. He was treated with topical antibiotics. After full resolution of keratitis, his condition was complicated by extensive corneal neovascularisation. A trial of photochemical corneal collagen CXL with riboflavin/ultraviolet A resulted in a dramatic improvement and resolution of the corneal neovascularisation. Thus, we can conclude that corneal collagen CXL could be a promising procedure to treat certain cases of extensive corneal neovascularisation.

\section{Keywords}

Corneal neovascularisation, collagen

crosslinking, keratitis, riboflavin

Disclosure: Mohammad M Shehadeh, Mohammad T Akkawi, Vasilios F Diakonis, Ammar A Aghbar and Abdelraheem Abu Shanab have nothing to declare in relation to this article. No funding was received in the publication of this article. The authors have no financial or proprietary interest in any materials or methods described herein.

Compliance with Ethics: All procedures were followed in accordance with the responsible committee on human experimentation and with the Helsinki Declaration of 1975 and subsequent revisions, and informed consent was received from the patient involved in this case study.

Authorship: All named authors meet the International Committee of Medical Journal Editors (ICMJE) criteria for authorship of this manuscript, take responsibility for the integrity of the work as a whole, and have given final approval to the version to be published.

open Access: This article is published under the Creative Commons Attribution Noncommercial License, which permits any non-commercial use, distribution, adaptation and reproduction provided the original author(s) and source are given appropriate credit.

Received: 24 June 2016

Accepted: 3 July 2017

Citation: European Ophthalmic Review,

2017;11(1):62-4

Corresponding Author: Mohammad M Shehadeh, Ophthalmology Department, An-Najah National University Hospital, An-Najah National University, Nablus, West Bank Palestine. E: mohammad.sh@najah.edu
Corneal neovascularisation is defined as extension of blood vessels into the avascular stroma. The most common diseases associated with corneal neovascularisation include infectious keratitis, stromal ulceration, inflammatory disorders, alkali burns, contact lens-related hypoxia, corneal graft rejection and limbal stem cell deficiency. ${ }^{1}$

Riboflavin/ultraviolet A (UVA) crosslinking (CXL) is a technique that was introduced several years ago for the treatment of keratectasia, keratoconus and infectious keratitis. ${ }^{2}$ Herein, we report a case of extensive corneal neovascularisation which was treated by ultraviolet corneal collagen CXL. Corneal collagen CXL could be an enormous hope in the treatment of corneal neovascularisation mentioning the limitations and undesirable side effects of the current treatment options for it. To our knowledge, this is the first report of using CXL to treat corneal neovascularisation.

\section{Case Report}

A 24-year-old man without known previous medical illnesses; his past ocular history included left eye penetrating corneal trauma, treated with a rotational flap 7 years prior to presentation. The patient was treated for 2 weeks as a case of herpetic keratitis in a private clinic with oral acyclovir $800 \mathrm{mg}$ five times daily, gatifloxacin $0.3 \%$ eye drops (E/D) every 2 hours (q2h) and atropine E/D q12h. There was no response to treatment and so was referred to our cornea clinic for further management.

On examination, the patient visual acuity (VA) was $1.0 \mathrm{OD}$, hand motion OS. Slit lamp examination revealed normal right eye, while a left eye examination showed purulent discharge, severely injected conjunctiva, central corneal abscess, corneal neovascularisation and $1 \mathrm{~mm}$ hypopyon. Lens and posterior segment were invisible, so B-scan ultrasonography was performed and revealed a clear vitreous with no signs of posterior segment involvement. His intraocular pressure (IOP) felt soft to touch. Corneal scraping was done and the specimen sent for Gram stain, Giemsa stain, culture and sensitivity. Acyclovir was discontinued because it was not a picture of herpetic keratitis and the history was free of previous herpetic eye disease. The patient was started on empirical treatment with fortified vancomycin $50 \mathrm{mg} / \mathrm{mL}$ q2h, fortified gentamicin $14 \mathrm{mg} / \mathrm{mL} \mathrm{q2h}$, resumed gatifloxacin E/D q6h and atropine q8h. Four days later the patient was re-examined; he was clinically improved with less eye pain, resolved hypopyon and better left eye VA (counting finger $50 \mathrm{~cm}$ ). Culture and stain were negative. However, because of the clinical improvement the same regimen was continued.

The patient was followed-up regularly afterwards. Full resolution of the abscess was noticed at the fourth week, but corneal clouding and extensive neovascularisation were present. The medications were discontinued and the patient started on lubricants only with regular follow-up. 
Figure 1: Coloured photograph shows significant corneal vascularisation and haze

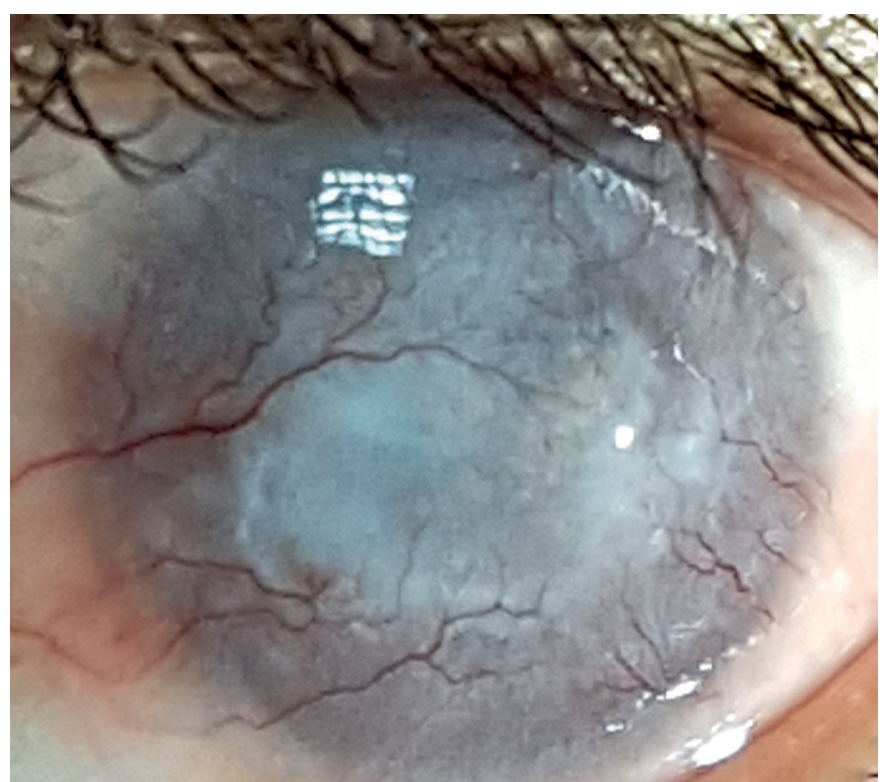

Four months later, VA improved to counting finger $1 \mathrm{M}$ OS, with no signs of infection, but the extensive corneal vascularisation remained the same (Figure 1).

The poor prognosis of corneal graft in the presence of such vascularisation was discussed with the patient as well as the limitations of other methods of corneal neovascularisation treatment.

The collagen CXL procedure, and the regression of some mild cases of corneal neovascularisation unintentionally observed in our cornea clinic after CXL procedure for other indications, was also discussed with him. He agreed and consented to undergo the procedure trial after being informed about the possible risks and side effects.

Standard collagen CXL procedure according to Wollensak et al. ${ }^{3}$ was performed. It involved anaesthetising the eye (with proxymetacaine hydrochloride $0.5 \%$ drops), removing the central $8-10 \mathrm{~mm}$ of the epithelium with a corneal brush and applying a riboflavin solution $(0.1 \%$ riboflavin-5-phosphate and 20\% dextran T-500) to the corneal surface 30 minutes before irradiation and at 5-minute intervals during the course of a 30-minute exposure to 370 nm UVA with an irradiance of $3 \mathrm{~mW} / \mathrm{cm}^{2}$. Bandage contact lens was placed for 5 days, and removed after observing the healing of the corneal epithelium. Gatifloxacin and dexamethasone E/D were administered for 1 week in addition to lubricant E/D.

One month later, the patient's vision improved to 0.05 OS and there was a dramatic improvement of the corneal neovascularisation (Figure 2). Three months post-CXL, vision improved to 0.3. VA and was 0.4 after the following 3 months with good patient satisfaction. One year later there was insignificant corneal vascularisation, a clearer cornea and the VA was 0.4 (Figure 3).

\section{Discussion}

Bevacizumab is a full-length, humanised murine monoclonal antibody that recognises all isoforms of vascular endothelial growth factor (VEGF). It is the first choice antiangiogenic therapy due to its availability and relatively low cost, with demonstrable efficacy when administered
Figure 2: Coloured photograph shows regression of corneal vascularisation 1-month postultraviolet corneal collagen crosslinking procedure

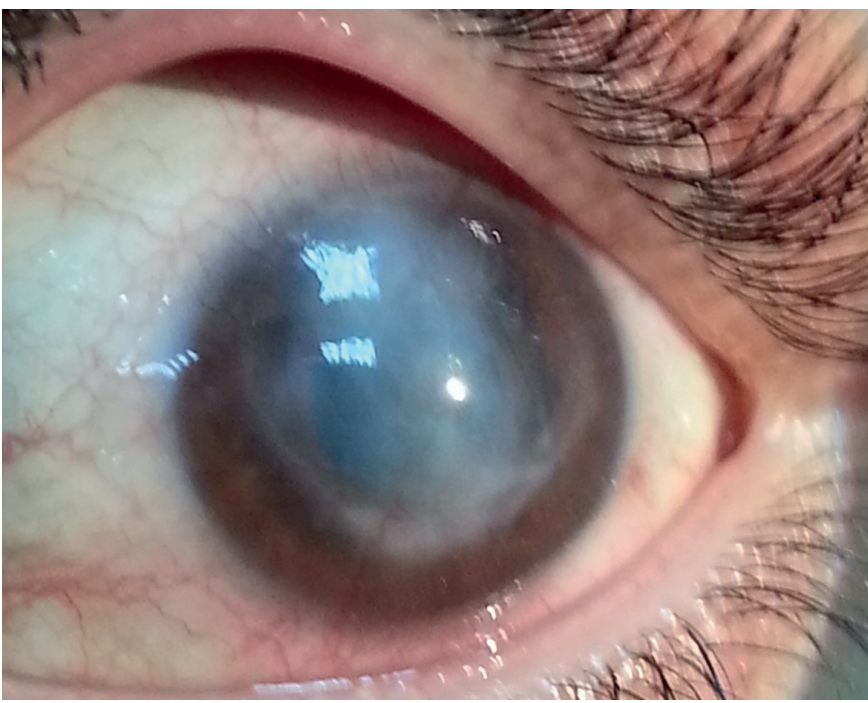

Figure 3: Coloured photograph shows nearly no corneal vascularisation and a clearer cornea 1-year postultraviolet corneal collagen crosslinking procedure

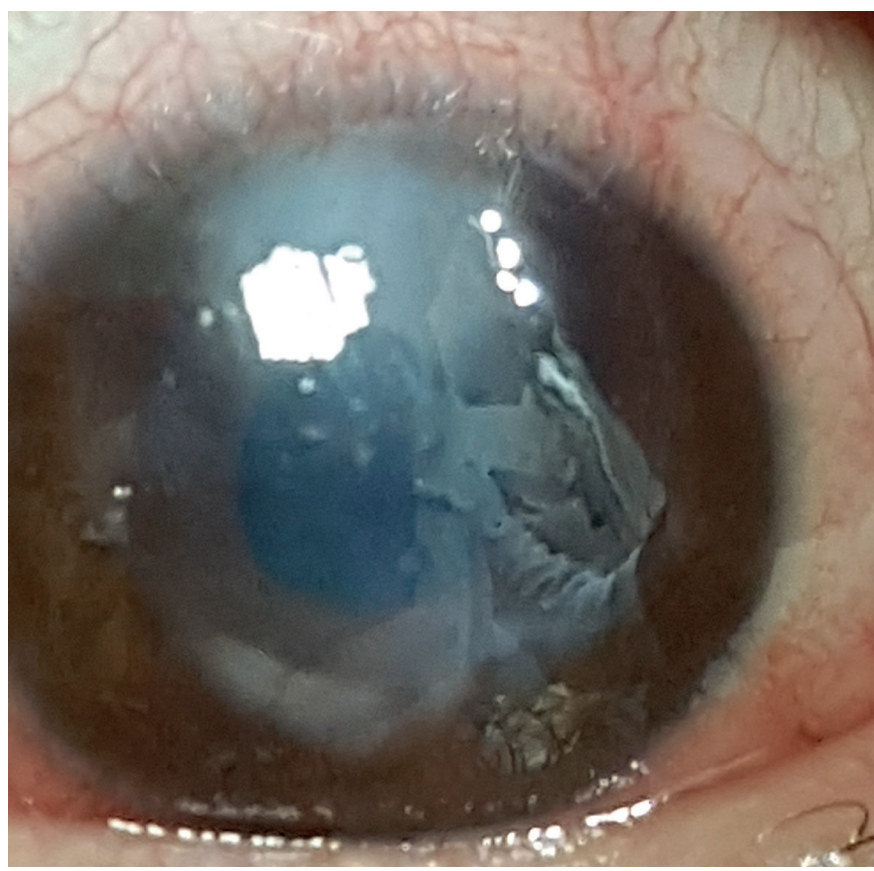

topically or subconjunctivally in reducing corneal neovascularisation. In addition, when corneal neovascularisation is due to limbal deficiency, ocular surface reconstruction using amniotic membrane graft or limbal stem cell transplantation is essential. ${ }^{4}$

Anti-VEGF antibody does have some limitations in treating corneal neovascularisation including its lower effect on deep vascularisation compared to superficial and active neovascularisation, which is necessary to repeat the treatment to maintain its positive effect over a period of time. ${ }^{5}$ Unfortunately, other current treatment options for corneal neovascularisation (steroid use, photodynamic therapy, laser photocoagulation and fine needle diathermy) have limited clinical efficacy with undesirable side effects. ${ }^{4}$ 
Photochemical CXL with riboflavin (vitamin B2)/UVA (370 nm) is a process mediated by photo-oxidation between UVA $(370 \mathrm{~nm})$ and riboflavin (vitamin B2).

Activation of riboflavin into triplet by UVA produces a reactive oxygen species (ROS) that reacts with the collagen fibres of the corneal stroma and results in forming new chemical bonds between its amino group that play the important rule in corneal mechanical strength enhancement. ${ }^{6}$

In psoriatic skin, local and systemic elevation of angiogenic cytokines including VEGF were observed, with fluctuation in line with disease activity. Treatments for psoriasis include psoralen and ultraviolet A (PUVA), and have been shown to inhibit angiogenesis and reduce levels of VEGF as part of their therapeutic effect. ${ }^{8}$ In vitro studies demonstrate that photochemotherapy with psoralen and UVA suppresses VEGF expression, inhibits angiogenesis and induces apoptosis of human endothelial cells. ${ }^{9}$ Vascular pathophysiological remodelling can be induced by excessive production of ROS or reducing its removal. These may include modification of the vascular wall, cytoskeletal structures, cellular attachments, composition of the extracellular matrix or apoptosis. ROS effects on apoptosis and vascular smooth muscle cells vary depending on the type and concentration of ROS and the amount of time the cell is exposed to the oxidant. ${ }^{10}$

We believe that corneal vascularisation regressed in our case due to the direct toxic effect of ultraviolet light on the vessels and indirectly by ROS. However, UVA may have exerted some antiangiogenic effect on the corneal blood vessels as in the case of psoriatic skin. In conclusion, corneal collagen CXL could be a promising procedure to treat certain cases of extensive corneal neovascularisation; however, more studies should be conducted to determine that the categories that would benefit from this approach and to assess its long-term safety.
1. Abdelfattah NS, Amgad M, Zayed AA, et al., Clinical correlates of common corneal neovascular diseases: A literature review, Int J Ophthalmol, 2015;8:182-93.

2. Zhang $X$, Tao $X C$, Zhang J, et al. A review of collagen cross-linking in cornea and sclera, J Ophthalmol, 2015:2015:289467.

3. Wollensak G, Spoerl E, Seiler T, Riboflavin/ultraviolet-A-induced collagen cross-linking for the treatment of keratoconus, Am J Ophthalmol, 2003;135:620-7.

4. Chang JH, Garg NK, Lunde E, et al., Corneal neovascularization:
An anti-VEGF therapy review, Surv Ophthalmol, 2012;57:415-29

5. Krizova D, Vokrojova M, Liehneova K, Studeny P, Treatment of corneal neovascularization using anti-VEGF bevacizumab, J Ophthalmol, 2014;2014:178132.

6. Raiskup F, Spoerl E, Corneal crosslinking with riboflavin and Raiskup F, Spoerl E, Corneal crosslinking with ribo
ultraviolet A. I. principles, Ocul Surf, 2013;11:65-74.

7. Young HS, Summers AM, Bhushan M, et al., Single-nucleotide polymorphisms of vascular endothelial growth factor in polymorphisms of vascular endothelial growth factor in
psoriasis of early onset, I Invest Dermatol, 2004;122:209-15.
8. Akman A, Dicle O, Yilmaz F, et al., Discrepant levels of vascular endothelial growth factor in psoriasis patients treated with PUVA, Re-PUVA and narrow-band UVB, Photodermatol Photoimmunol Photomed, 2008;24:123-7.

9. Deng $\mathrm{H}$, Yan $\mathrm{CL}$, $\mathrm{Hu}$ Y, et al., Photochemotherapy inhibits angiogenesis and induces apoptosis of endothelial cells in vitro,
Photodermatol Photoimmunol Photomed, 2004;20:191-9.

10. Staiculescu MC, Foote C, Meininger GA, Martinez-Lemus $L A$, The role of reactive oxygen species in microvascular remodeling, Int J Mol SCi, 2014;15:23792-835. 